\title{
Air travel-related symptomatic deep venous thrombosis in cruise ship passengers
}

\author{
Wojciech S. Pietrzyk
}

Anaesthesiology and Intensive Therapy Department, St. Alexander Hospital, Kielce, Poland

\begin{abstract}
Background: Long air travel is a risk factor for deep venous thrombosis (DVT). The aim of the study was to report the occurrence of symptomatic DVT cases in cruise ship passengers after long haul flights and to discuss applied diagnostic methods.

Materials and methods: A retrospective analysis of medical records of adult patients diagnosed with DVT in medical facility of the passenger ship was performed. On the basis of clinical examinations, B-mode ultrasound scans of the lower extremity venous systems, international normalised ratio (INR) tests, working diagnoses of DVT were established. The Wells score was used for stratification. Within 4-48 h of the diagnosis, D-dimer tests in blood, ultrasound Duplex examinations and specialists' consultations of DVT suspected patients were performed ashore.

Results: A study showed $3(0.15 \%)$ patients suspected of DVT of a total number of 2,007 passengers who have completed a flight $>8 \mathrm{~h}$ in the analysed period. The medial time from the embarkation to the onset of symptoms was $68.7 \mathrm{~h}$. Based on the Wells DVT score, in $2(0.1 \%)$ patients the probability of DVT was determined to be likely. Both the ultrasound examinations and D-dimer tests were positive. Those patients were diagnosed by shore specialists as DVT. One (0.05\%) patient determined as DVT unlikely according to the Wells scale, her INR indicated hypercoagulable state, but Duplex scan as well D-dimer test were negative and DVT suspicion was excluded.

Conclusions: A clinical suspicion of DVT among passengers who joined the ship after their long haul flights could be an important diagnostic problem for ship's doctors. The Wells scale allows to stratify the risk, but to rule out suspicion of DVT, a negative result of D-dimer test must be demonstrated. A portable ultrasound device helps in onboard clinical evaluation of DVT suspected patients. Due to possible fatal complications of DVT which can lead to pulmonary embolism and cause medico-legal issues, a definitive diagnosis should only be obtained in cooperation with land-based health care providers.
\end{abstract}

(Int Marit Health 2016; 67, 2: 66-71)

Key words: deep venous thrombosis, venous thromboembolism, air flight, cruise ship, maritime medicine

\section{INTRODUCTION}

For the first time the association between deep venous thrombosis (DVT) and long air flight was observed in a 54-year-old medical doctor who took a 14-h-flight from Boston to Caracas. The case study was published more than half a century ago by a surgeon John Homans, widely known for the description of symptoms indicative of thrombosis in the leg veins [1]. Since then long haul air travel has been considered a risk factor for DVT, including pulmonary embolism (PE), which is potentially fatal.
Although today, from the medical point of view, sea voyage is a safe way of travelling, a particular group of cruise ships passengers who embark the vessel after a long haul flight are vulnerable to DVT. They travel across several time and climate zones; also most of them are at an older age - this may be associated with a higher incidence of diseases, which additionally increase the risk of venous thromboembolism (VTE). Limited resources for the diagnosis and treatment of patients with VTE on board a cruise ship poses a significant challenge to the ship's doctor. The aim 
Table 1. Demographic data

\begin{tabular}{lllll}
\hline Number of passengers long haul flight $>\mathbf{8} \mathbf{h}$ & Female & Male & Mean age [years] \\
\hline Total & 2,007 & $1,087(54.16 \%)$ & $920(45.84 \%)$ & $68.5(23-87)$ \\
Deep venous thrombosis suspicion & 3 & $2(66.67 \%)$ & $1(33.33 \%)$ & $69(65-74)$
\end{tabular}

Table. 2 Wells score (clinical model for predicting the pretest probability of deep vein thrombosis)

\begin{tabular}{lc}
\hline Symptom/Present & Score \\
\hline Active cancer (patient receiving treatment for cancer within the previous 6 months or currently receiving palliative treatment) & 1 \\
Paralysis, paresis or recent plaster immobilization of the lower extremities & 1 \\
Recently bedridden for 3 days or more, or major surgery within the previous 12 weeks requiring general or regional anaesthesia & 1 \\
Localised tenderness along the distribution of the deep venous system & 1 \\
Entire leg swollen & 1 \\
Calf swelling at least 3 cm larger than that on the asymptomatic side (measured 10 cm below tibial tuberosity) & 1 \\
Pitting oedema confined to the symptomatic leg & 1 \\
Collateral superficial veins (non varicose) & 1 \\
Previously documented deep-vein thrombosis & 1 \\
Alternative diagnosis at least as likely as deep-vein thrombosis & $(-) 2$
\end{tabular}

Unlikely $=$ Wells score $<2$; Likely $=$ Wells score $\geq 2$

of the study was to assess the occurrence of symptomatic DVT cases and the current protocol of the DVT diagnosis in the population of passengers embarking on a cruise ship after long haul flights.

\section{MATERIALS AND METHODS}

A retrospective study of medical records of adult patients treated at the Ship's Medical Centre (SMC) on a passenger vessel cruising in the sub-tropical and tropical regions, during the winter seasons of 2013 and 2014, was performed. The clinical data of patients who presented with a suspicion of DVT after an air flight which lasted more than $8 \mathrm{~h}$ were analysed. The total number of cruise ship passengers who have completed flight $>8 \mathrm{~h}$ in the above - mentioned period was 2,007. The vast majority of the passengers were adult Caucasians - who took long-distance flights $12 \mathrm{~h}$ before embarking the ship. Typically, a cruise lasts 2 or 3 weeks, but some passengers stay on a cruise ship longer. The study did not include crew members because of the difference in demography and the type of activities performed on board [2]. Demographic data are presented in Table 1. The working diagnosis of DVT was established on the basis of a patient's history and physical examination. The clinical model for assessing the pretest probability of DVT, known as the Wells score (Table 2) was used for risk stratification [3]. Additionally an initial B-mode ultrasound examination of the lower ex- tremity venous system, using a portable ultrasound device (Aloka SSD500, convex 3.5 MHz) was performed by ship's doctor at the SMC. The technique of 2-point compression ultrasonography examination of the common femoral and popliteal veins was employed. In order to assess the state of coagulation in the DVT suspected patients, an international normalised ratio (INR) test using handheld device (CoaguCheck XS INR System, Roche) was performed in the SMC laboratory.

Due to some legal regulations and the company policy, final confirmation/exclusion of DVT was provided by a consultant vascular surgeon or an emergency specialist from a hospital on shore (an onshore hospital). We also required specialist radiologists ashore for Duplex ultrasound examination of the affected lower leg and to carry out a study of D-dimer in blood. The D-dimer test result was considered within range if the level was $<500 \mathrm{mcg}$ FEU/I or $250 \mathrm{mcg}$ DDU/I or it was within reference values accepted by the laboratory on shore. Diagnostic procedures and consultations on shore were performed within $4-48 \mathrm{~h}$ of the DVT. In cases when the consultations could not be carried out within one day, therapeutic doses of low molecular weight heparins were administered. One patient diagnosed with DVT, relating to immobilization in plaster due to a lower leg injury was excluded from the study. The percentage figures presented in tables have been rounded to the second decimal place. 
Table 3. Clinical data of symptomatic cases of deep venous thrombosis (DVT)

\begin{tabular}{|c|c|c|c|c|c|c|c|}
\hline \multirow[t]{2}{*}{ No. } & \multicolumn{4}{|l|}{ Examination on board } & \multicolumn{3}{|c|}{ Examination ashore } \\
\hline & $\begin{array}{l}\text { Onset of symptoms after } \\
\text { embarkation [h] }\end{array}$ & Wells score & $\begin{array}{l}\text { B-mode } \\
\text { US-scan }\end{array}$ & INR & D-dimers & $\begin{array}{l}\text { Duplex } \\
\text { US-scan }\end{array}$ & $\begin{array}{l}\text { Consultation/ } \\
\text { /DVT confirmed }\end{array}$ \\
\hline 1 & 38 & 1 & $(+/-)$ & 0.75 & $(-)$ & $(-)$ & $(-)$ \\
\hline 2 & 72 & 3 & $(+)$ & $\mathrm{N}$ & $(+)$ & $(+)$ & $(+)$ \\
\hline 3 & 96 & 2 & $(+)$ & $\mathrm{N}$ & $(+)$ & $(+)$ & $(+)$ \\
\hline
\end{tabular}

(+) positive; (-) negative; (+/-) non-conclusive; N - normal; INR - international normalised ratio; US - ultrasonography

Table 4. Results of diagnostic evaluations of symptomatic deep venous thrombosis cases

\begin{tabular}{lll}
\hline Deep venous thrombosis & Number of cases & Percentage \\
\hline Suspicion (history + on board examination) & 3 & $0.15 \%$ \\
Unlikely (Wells score $<2$ ) & 1 & $0.05 \%$ \\
Likely (Wells score $\geq 2$ ) & 2 & $0.1 \%$ \\
Confirmed (ashore examination) & 2 & $0.1 \%$
\end{tabular}

\section{RESULTS}

Clinical suspicion of DVT was established in 3 patients, which is approximately $0.15 \%$ of all passengers taking long distance flight. All 3 patients had a history of additional risk factors of DVT and the physical examination showed the presence of symptoms suggestive a of pathology in the lower limb venous system. The medial time from the embarkation to the onset of symptoms was 68.6 hours.

Based on the Wells score in $2(0.1 \%)$ patients the probability of DVT was determined to be likely and in 1 (0.05\%) unlikely. One patient with a family history of VTE, had lowered INR (INR = 0.75) which suggested hypercoagulable state. The rest of the patients had INR values within normal limits (0.80-1.20). An initial onboard B-mode ultrasound examination of the lower extremity venous system confirmed the presence of thrombi in popliteal veins in 2 patients, and in the case of 1 patient the test was inconclusive (difficult examination conditions due to obesity). Duplex ultrasonography performed by radiologists ashore - confirmed the presence of thrombi in the lower extremity venous system in 2 patients and excluded the presence of a thrombus in the patient with inconclusive $B$ mode result. D-dimer levels were elevated in 2 patients, while in 1 patient they were in normal range. Shore consultant physicians confirmed DVT diagnosis in $2(0.1 \%)$ patients and in 1 case superficial thrombophlebitis in varicose veins was diagnosed. In the analysed period no cases of PE were observed. Clinical data and results are presented in Tables 3 and 4.

\section{DISCUSSION}

The presence of adult population after long-haul flights travelling on board a passenger ship for at a least 2-week period gives a unique opportunity for observation of symptomatic DVT prevalence.

Unfortunately the diagnostic tools for the detection and confirmation of DVT, normally available in most hospitals, are very limited in ship's medical facilities. The factors which could limit our study are different laboratory methods, equipment or specialists in shore hospitals that cooperated during diagnostic process of passengers with suspected DVT.

The present epidemiological data about the occurrence of DVT after long haul flights vary widely depending on thromboembolic location and the fact whether the thrombosis is symptomatic or asymptomatic. A well designed prospective study of Schwartz et al. [4] reported the incidence of isolated calf muscle venous thrombosis (ICMVT) in $2.1 \%$ of 964 passengers after long haul flights. According to the authors, the ICMVT in the soleus and gastrocnemius muscles was considered as a precursor or the first step toward DVT, but only one in all 20 cases with ICMVT was symptomatic. The same study showed $0.7 \%$ occurrence of DVT. Among DVT patients only 2 cases were symptomatic. Furthermore, one case of PE was diagnosed by ventilation-perfusion lung scan. The authors concluded that long haul flights of $8 \mathrm{~h}$ and longer double the risk for ICMVT [4].

Another study reported that after air travel lasting more than $8 \mathrm{~h}, 10 \%$ of passengers, who were not wearing compression stockings, developed asymptomatic DVT in the calf. The occurrence of DVT was documented by detailed Duplex ultrasonography examinations of lower limbs, performed before long-haul flights and within $48 \mathrm{~h}$ after landing. None of the passengers wearing compression stockings had DVT; however, $3 \%$ of them developed superficial thrombophlebitis in varicose veins [5]. 
In our research we observed symptomatic DVT in $2(0.1 \%)$ cases. It is difficult to compare the results of well-designed prospective research with our simple case observational retrospective study. However, taking into consideration the incidence of purely symptomatic DVT cases only, the difference between our results $(0.1 \%)$ and the ones cited above $(0.0-0.2 \%)$ does not seem distinct. The observed lower incidence of symptomatic DVT among a cruise ship passengers could be explained by the fact that passengers with pre-existing vascular diseases and known risk factors of VTE did not expose themselves for long haul flights as precondition for the cruise. It is also possible that patients with less presented symptoms of DVT did not search for chargeable medical service in the SMC. Some other non-medical factors, such as the consultation fee or differences in insurance systems can influence the patient's decision to look for medical assistance [6].

The process of adherence and organisation of the venous thrombus does not begin until 5-10 days after its formation. Because of this, PE and DVT can coexist and then they are called a VTE. According to some authors asymptomatic DVT is found in 40-50\% patients with PE while 40\% patients with DVT have clinically silent PE [7]. In our study, we did not diagnosed PE, but it is possible that some silent or low symptomatic PE cases could be underdiagnosed during a clinical examination.

There are several medical reports of passengers with severe PE on arrival at major airports. The risk of this potentially fatal complication of DVT seems to be very low and is estimated as a 1.65 event per 1 million passengers, providing the flight was longer than $8 \mathrm{~h}$. Only about one-third of the patients with travel associated PE presented with signs of DVT $[8,9]$. Limitation of those studies was rather short period of observation of PE occurrence. Only those cases of PE observed during the flight and very shortly after arrival were reported. Also, passengers who died during the flight or at the airport, with PE being the possible cause of death, were not taken into account.

The only available data on VTE cases on board cruise ships is derived from a study of Prina et al. [10]. In his analysis of 104 critically ill, evacuated patients, 4 (3.8\%) were initially diagnosed with PE/DVT. This study, however, does not provide much information about the possible association between PE and long haul flights or other risk factors of VTE.

It is widely accepted that age over 65 , pregnancy, oestrogen-progestin hormonal treatment, congestive heart failure, obesity, smoking or known thrombophilia are risk factors for VTE [11]. Additionally, height $<165 \mathrm{~cm}$ or $>$ and $185 \mathrm{~cm}$ and body mass index $>25 \mathrm{~kg} / \mathrm{m}^{2}$ in regard to a standard passenger seat size could be air travel related risk factors [12].

A thorough clinical examination and careful observation of a patient is the most common and readily available diag- nostic method for a of ship's doctor. But no single physical manifestation, even a very suggestive one, is sufficient to establish diagnosis of DVT. The most specific symptom is unilateral or bilateral oedema of the limb. Thrombus that affects the proximal veins produces leg oedema. Calf pain, which is present in $50 \%$ of cases, is not specific for DVT. The classic Homans' sign is neither sensitive nor specific - as it is present in less than one-third of patients with DVT confirmed by venography, and is found in more than $50 \%$ of patients without DVT [13].

The Wells DVT score provides valuable clinical support during complex diagnostic evaluation. The pretest probability score calculated with this tool can be stratified into either 2 or 3 risk groups. In the 3-risk group model, patients with a score of 0 or less are considered low risk patients, the score of 1-2 presents moderate risk, and the score of 3 or more is classified as high risk, with $5 \%, 17 \%$ and $53 \%$ probability of DVT respectively. In the more frequently used 2-risk group evaluation, patients are stratified as DVT unlikely (the Wells score < 2) or DVT likely (the Wells score $\geq 2$ ) where probability of DVT is $6 \%$ and $28 \%$ respectively $[14,15]$. By combining this score with the results of a highly sensitive D-dimer assay and proximal leg vein ultrasound scan further evaluation is possible [16].

The diagnostics of DVT is one of the core emergency ultrasound applications indicated by American College of Emergency Physicians [17]. Duplex ultrasound examination is currently the first line imaging diagnostic method performed to detect or visualise a thrombus in the venous system. It consists of two parts, first, a B-mode ultrasound examination to obtain an image/picture and second, to evaluate vein flow using Doppler effect. Due to limitations of our portable ultrasound device we could only use the B-mode 2-point compression method for DVT diagnosis. According to some authors, compression ultrasound, conducted by physician in the Emergency Room is enough to detect the presence or absence of proximal lower extremity DVT. Depending on the author, the sensitivity and specificity of this method ranged from $70 \%$ up to $100 \%$ and $89 \%$ up to $99.4 \%$, respectively $[18,19]$. In our study, the Duplex ultrasound examination performed by a specialist of radiology in shore hospitals generally confirmed our clinical impressions after B-mode ultrasonography examination on board the ship. There is not enough data to perform reliable statistical analysis, but a figurative quote that' a portable emergency ultrasound could be a stethoscope extension' is very appropriate in ship's doctor practice. Additionally the telesonography a form of telemedicine, which is capable of transferring the images to reference centres, can provide valuable medical interpretations and possible clinical answers [20, 21].

All of our patients with the clinical suspicion of DVT were tested for D-dimer levels in shore laboratories. As shown 
in Table 3 only one case was negative which allowed us to rule out the DVT diagnosis. The D-dimer tests that shore laboratories used for 2 patients were a rapid enzyme-linked immunoabsorbent assay (ELISA). In 1 case of a positive result we did not receive any information about the method of the test that was used, which is a limitation of our study. There are commercially available rapid test kits for the detection of D-dimers in blood, which could improve diagnostic process on board in VTE suspected patients. Pulmonary embolism is a potentially fatal complication of DVT; it typically requires antithrombotic or thrombolytic treatment and medevac. It is also an important factor in a differential diagnosis of many life threatening conditions, including acute chest pain, which can create diagnostic difficulties for medical personnel on board [22]. Therefore, it seems reasonable to consider the usefulness of rapid D-dimer tests in medical facilities on board cruise ships.

We also requested Duplex ultrasound scans from cooperating shore hospitals for all our patients despite their D-dimer tests results and Wells scores. Patients classified as DVT unlikely (Wells score < 2) and negative D-dimer test could have been "overdiagnosed" which was due to their personal petition and the requirements and conditions of Travel Insurances, as well as the ship's doctor good practice to have fully diagnosed patients on board before sailing away.

Unfortunately, the Wells score system has its limitations as not all thrombophilia-related factors are reflected in the classification. An important risks factor for VTE are congenital, acquired and mixed excessive blood clotting disorders. It is estimated that up to $20 \%$ of the total population may have some degree of increased clotting tendency. Among the inherited disorders, the most important are gene mutations of factor V (factor V Leiden, FVL), variant G20210A of prothrombin gene, genetic deficiency of antithrombin III, protein $\mathrm{C}$ and $\mathrm{S}$. While antiphospholipid syndrome or hyperhomocysteinaemia are the most essential of acquired disorders [23, 24]. Although widely accepted PREP-Health Care Guidelines for Cruise Ship Medical Facilities of ACEP [25] do not impose the requirement of having laboratory coagulation testing on board, on most cruise ships it is possible to perform INR test due to the fact that a significant number of passengers take oral antivitamin $\mathrm{K}$ anticoagulants. We used this coagulation test, the only one available on board, as an adjunct to assess a patient's coagulation state related to tissue factor pathway (extrinsic) of the coagulation cascade. This is not a standard procedure, but it could support our diagnostic process on board. According to recommendations, testing for hypercoagulable states should be performed in stages and due to its complexity and high costs in cases of idiopathic or recurrent VTE only [26].

\section{CONCLUSIONS}

A clinical suspicion of DVT among passengers who joined the cruise ship after their long haul flights could be an important diagnostic problem for the ship's doctor shortly after embarkation.

The Wells scale allows stratifying the risk; however, to rule out the suspicion of DVT it is necessary to perform at least a blood test for D-dimers.

Portable ultrasound, preferably with Doppler option, could be an useful diagnostic tool during clinical evaluation of patients with suspected DVT on board the ship.

The definitive diagnosis of DVT should only be made in cooperation with land-based health care providers due to its potentially fatal complications, including PE, its complex pathophysiology and medico-legal issues as well.

Further prospective studies concerning incidence of air travel-related DVT in cruise ships passengers are needed.

\section{REFERENCES}

1. Homans J. Thrombosis of the deep leg veins due to prolonged sitting. N Engl J Med 1954; 250: 148-149.

2. Pietrzyk W. The Role of Emergency Medicine In Ship's Doctor Practice. XIII Winter School of Emergency Medicine, 2004 Karpacz, Poland; Materials of International Symposium “Progress In Emergency Medicine", p. 176-177.

3. Wells PS, Anderson DR, Rodger M et al. Evaluation of D-dimer in the diagnosis of suspected deep-vein thrombosis. N Engl J Med 2003; 349: 1227-1235.

4. Schwartz T, Siegert G, Oettler W et al. Venous thrombosis after long-haul flights. Arch Intern Med 2003; 163: 2795-2764.

5. Scurr JH, Machin SJ, Bailey-King S, Mackie IJ, McDonald S, Smith PD. Frequency and prevention of symptomless deep-vein thrombosis in long-haul flights: a randomized trial. Lancet 2001; 357: 1485-1489.

6. Tomaszewski R, Nahorski LW. Interpopulation study of medical attendance aboard a cruise ship. Int Marit Health 2008; 59: 1-4.

7. Turkstra F, KuijerPMM, van BeekEJR,Brandjes DPM, Buller HR, Cate JW. Diagnostic utility of ultrasonography of leg veins in patients suspected of having pulmonary embolism. Ann Intern Med 1997; 126: 775-781.

8. Perez-Rodriguez E, Jimenez D, Diaz G et al. Incidence of air travel -related pulmonary embolism at the Madrid-Barajas airport. Arch Intern Med 2003; 163: 2766-2770.

9. Lapostolle F, Surget V, Borrow SW et al. Severe pulmonary embolism associated with air travel. N Engl J Med 2001; 345: 779-783.

10. Prina LD, Orazi UN, Weber RE. Evaluation of emergency evacuation of critically ill patients from cruise ships. J Travel Med 2001; 8: 285-292.

11. Kahn SR, Lim W, Dunn AS et al. Prevention of VTE in nonsurgical patients: antithrombotic therapy and prevention of thrombosis. 9th Ed. American College of Chest Physicians. Evidence-Based Clinical Practice Guidelines. Chest 2012; 141 (2 suppl.): e195S-e226S.

12. Bartholomew JR, Schaffer JL, McCormick GF. Air travel and venous thromboembolism: Minimizing the risk. Cleve Clin J Med 2011; 78: 111-120.

13. Gorman WP, Davis KR, Donnelly R. ABC of arterial and venous disease. Swollen lower limb-1: general assessment and deep vein thrombosis. BMJ 2000; 320: 1453-1456.

14. Hargett CW, Tapson VF. Clinical probability and D-dimer testing: how should we use them in clinical practice? Semin Respir Crit Care Med 2008; 29: 15-24. 
15. Bates SM, Jaeschke R, Stevens SM et al. Diagnosis of DVT: Antithrombotic Therapy and Prevention of Thrombosis, 9th ed: American College of Chest Physicians Evidence-Based Clinical Practice Guidelines. Chest 2012; 141 (2 suppl.): e351S-e418S.

16. Venous thromboembolic diseases: the management of venous thromboembolic diseases and the role of thrombophilia testing, Issued June 2012. National Institute for Health and Clinical Excellence Web site. Available at: http://www.nice.org.uk/guidance/cg144. Accessed: January 21, 2016.

17. American College of Emergency Physicians. Policy Statement: emergency ultrasound guidelines. Ann Emerg Med 2009; 53: 550-570.

18. Kline JA, O'Malley PM, Tayal VS, Snead GR, Mitchell AM. Emergency clinician-performed compression ultrasonography for deep venous thrombosis of the lower extremity. Ann Emerg Med 2008; 52: 437-445.

19. Crisp JG, Lovato LM, Jang TB. Compression ultrasonography of the lower extremity with portable vascular ultrasonography can accurately detect deep venous thrombosis in the emergency department. Ann Emerg Med 2010; 56: 601-610.

20. Linping $P$, Gillman LM, McBeth PB et al. Potential Use of Remote Telesonography as a Transformational Technology in Underreso- urced and/or Remote Settings. Emerg Med International 2013, Article ID 986160.

21. Nikolic N, Mozetic V, Modrcin B, Jaksic S. Might telesonography be a new useful diagnostic tool aboard merchant ships? A pilot study. Int Marit Health 2006; 57: 198-207.

22. Dahl E. Acute chest pain in cruise ships. Int Marit Health 2015; 66: 4-5.

23. Abramson N, Abramson S. Hypercoagulability. Clinical Assessment and Treatment. South Med J 2001; 94: 1013-1020.

24. Bande BD, Bande SB, Mohite S. The hypercoagulable states in anaesthesia and critical care. Indian J Anaesth 2014; 58: 665-671.

25. American College of Emergency Physicians Web site. Available at: http://www.acep.org/Physician-Resources/Clinical/PREP-Health-Care-Guidelines-for-Cruise-Ship-Medical-Facilities. Accessed: January 21, 2016.

26. Deitcher S. Hypercoagulable states, hematology and oncology, text based CME cases. Web site Cleveland Clinic, Center For Continuing Education. Available at: http://www.clevelandclinicmeded.com/ medicalpubs/diseasemanagement/hematology-oncology/hypercoagulable-states. Accessed: January 10, 2016. 